\title{
Trajectories of the infinitesimal mass around the triangular equilibrium points in elliptical restricted three bodies problem under oblate and radiating primaries for the binary systems
}

\author{
Nutan Singh $^{1 *}$, A Narayan ${ }^{2}$, B Ishwar ${ }^{3}$ \\ ${ }^{1}$ Dept. of Mathematics, Rungta college of Engg \&Tech., Bhilai, 490020 \\ ${ }^{2}$ Dept. of Mathematics ,Bhilai Institute of Technology, Durg, 491001 \\ ${ }^{3}$ Principal Investigators, D ST, New Delhi, B.R.A BiharUniversity Muzaffarpur \\ *Corresponding author E-mail: ashutoshmaths.narayan@gmail.com
}

\begin{abstract}
This paper describes the trajectory of the infinitesimal mass around $\mathrm{L}_{4}$ of the triangular equilibrium points for the binary systems in the elliptical restricted three body's problem (ERTBP), where both oblate primaries are radiating. The solutions for the perturbed motion in the vicinity of $\mathrm{L}_{4}$ is given by $\mathrm{u}(\mathrm{f})$ and $\mathrm{v}(\mathrm{f})$ function. The stability of the infinitesimal mass around the triangular points is also studied by plotting $\mathrm{u}(\mathrm{f})$ and $\mathrm{v}(\mathrm{f})$ curve. It is found that radiation pressure, oblateness and eccentricity show a significant effect on the trajectory and stability of the infinitesimal mass around the triangular equilibrium points. Simulation technique has been used to design the trajectory of the binary systems (Achird, Luyten, $\alpha$ Cen AB, Kruger-60 and Xi-Bootis).
\end{abstract}

Keywords: Radiation Pressure; Triangular Points; Oblateness Parameters; Trajectory of Infinitesimal Stability.

\section{Introduction}

In the three-body problems, three bodies move in space under their mutual gravitational interactions, as described by Newton's theory of gravitation. In general the motions of the bodies take place in three dimensions, and there are no restrictions on their masses or on the initial conditions. Since, the motion of the three bodies are in general unpredictable, it makes the three -body problems, as one of the most challenging problems in the history of science. The problems adapted to the need of time are the restricted three bodies problem (RTBP), in which the third body has no influence on the other two bodies. RTBP is classified into circular RTBP and ERTBP depending upon the motion in, circular or elliptic orbits respectively. The ERTBP is a generalization of classical problem; where the primaries move in the elliptical orbits about their common centre of mass, uninfluenced by the third body having negligible mass. The RTBP assumes that the masses of the primaries are spherically symmetrical in homogenous layer, but it is found that the celestial bodies are sufficiently oblate which produce deviations from the two-body problems; inspiring several researchers to include oblateness of the primaries in their studies of RTBP. It is well known that there exist five relative equilibrium solutions; three of them are collinear, denoted by L1, L2, L3 and the other two are triangular, denoted by L4 and L5. The equilibrium points that appear in RTBP are very important for astronautical applications as space station can be easily located at these points since the consumption of fuel required for station keeping is less.
SubbaRao \& Sharma (1975) considered the primary as oblate spheroid and proved that the range of the mass parameter leading to stable solution decreases due to oblateness. Sharma (1982) investigated the linear stability of the triangular points where the more massive primary is a source of radiation and oblate spheroid as well. Kumar \& Ishwar (2011) studied the motion of the infinitesimal in generalized photo gravitational ERTBP, considering oblate spheroid. Singh and Umar (2012b) studied the stability of the triangular points in ERTBP, where both bodies emit light energy simultaneously and found that the range of stability varies with change in eccentricity, oblateness and radiation pressure.

The ERTBP has been described in considerable details by Ammar(2008), Danby (1964), Szebehely(1967), Markeev (1978), Selaru-Cucu (1994), Halan and Rana (2001). The influence of the eccentricity of the orbits of the primaries with or without radiation pressure on the existence and stability of the equilibrium points are studied by Erdi (2009), Gyorgrey (1985), Grebenikov (1964), Kumar and Choudhary (1986), Markeellos (1992), Roberts (2002), Floria (2004), Zimvoschikov and Thakai (2004), Ammar (2008), Singh and Umar (2012a, 2012b), Narayan and Singh (2014 a, b,c). Singh and Umar (2012b) studied the locations of the triangular points considering oblate and radiating primaries for the binary system and found that their position are significantly affected by the eccentricity of the orbits, oblateness and radiation factors. They studied the stability of the triangular points in general by varying radiations pressure, semi-major axis and oblateness parameter and investigated that the size within the region of stability decrease with increase in the values of the parameter involved. This work is extension of the work of Singh and Umar (2012b) by designing a trajectory of the infinitesimal mass around the triangu- 
lar equilibrium points for binary systems (Achird, Luyten, $\alpha$ Cen $\mathrm{AB}$, Kruger-60 and Xi-Bootis).

This paper describes the trajectory and analyzes the stability of the infinitesimal mass around $\mathrm{L}_{4}$ of the triangular equilibrium points in ERTBP considering oblate and radiating primaries for the binary systems. The solutions are found by plotting $u$ (f) and $v$ (f) curves for binary systems: (Achird, Luyten, $\alpha$ Cen AB, Kruger-60 and Xi-Bootis). The stability of the infinitesimal is studied by exploiting simulation technique and by drawing different trajectory for the binary systems. For numerical calculations, we consider our model of the (ERTBP) to be composed of the binary system (Achird, Luyten, $\alpha$ Cen AB, Kruger-60 and Xi-Bootis) as primaries and the infinitesimal mass as a space craft.

This paper is organized as: Section 1 gives introduction, Section 2 determines the equations of motion; Sections 3 establishes stability of the triangular equilibrium points, Section 4 and Section 5 are trajectories of the infinitesimal mass around the triangular equilibrium points and discussion $\&$ conclusion respectively.

\section{Equations of motion}

The equations of motion of the infinitesimal mass in dimensionless units in a rotating pulsating coordinate system following Singh and Umar (2012 a, 2012b) is given as:

$$
\begin{aligned}
& \xi^{\prime \prime}-2 \eta^{\prime}=\Omega_{\xi^{*}}^{*}, \\
& \eta^{\prime \prime}+2 \xi^{\prime}=\Omega_{\eta}^{*}, \\
& \zeta^{\prime \prime}=\Omega_{\zeta}^{*}
\end{aligned}
$$

Where, $\Omega$ is the force function defined as:

$$
\begin{aligned}
& \Omega=\left(1-e^{2}\right)^{-1 / 2} \times \\
& {\left[\frac{\xi^{2}+\eta^{2}}{2}+\frac{1}{n^{2}}\left(\begin{array}{l}
\frac{(1-\mu) q_{1}}{r_{1}}+\frac{\mu q_{2}}{r_{2}}+ \\
\left.\frac{(1-\mu) A_{1} q_{1}}{2 r_{1}^{3}}+\frac{\mu A_{2} q_{2}}{2 r_{2}^{3}}\right)
\end{array}\right) .\right.}
\end{aligned}
$$

The mean motion, $\mathrm{n}$ is given by

$$
n^{2}=\frac{\left(1+e^{2}\right)^{1 / 2}\left(1+\frac{3 A_{1}}{2}+\frac{3 A_{2}}{2}\right)}{a\left(1-e^{2}\right)},
$$

And

$$
r_{i}^{2}=\left(\xi+\xi_{i}\right)+\eta^{2}+\zeta^{2}, i=1,2
$$

$\xi_{1}=-\mu, \xi_{2}=1-\mu, \mu=\frac{m_{2}}{m_{1}+m_{2}}$.

Here, $\mathrm{m}_{1}, \mathrm{~m}_{2}$ are the masses of the bigger and smaller primary, located at the points $\left(\xi_{i}, 0,0\right), i=1,2 ; \mathrm{q}_{1}$ and $\mathrm{q}_{2}$ are the mass reduction factors. $A_{1}$ and $A_{2}$ are the oblateness parameters; $r_{i},(i=1,2)$ are the distances of the infinitesimal mass from the bigger and smaller primaries and ' $a$ ' and ' $e$ ' are the semimajor axis and eccentricity of the orbits.

The triangular equilibrium points denoted by $L_{4,5}(\xi, \pm \eta)$ as given by Singh and Umar (2012b) is:

$$
\begin{aligned}
& \xi= \frac{1}{2}-\mu+\frac{1}{2}\left(a q_{1}\right)^{2 / 3}\left(1-e^{2}-A_{1}-A_{2}+A_{1}\left(a q_{1}\right)^{-2 / 3}\right) \\
&-\frac{1}{2}\left(a q_{2}\right)^{2 / 3}\left(1-e^{2}-A_{1}-A_{2}+A_{2}\left(a q_{2}\right)^{-2 / 3}\right), \\
& \eta= \pm\left[\begin{array}{l}
\left(a q_{1}\right)^{2 / 3}\left(1-e^{2}-A_{1}-A_{2}+A_{1}\left(a q_{1}\right)^{-2 / 3}\right)- \\
\frac{1}{4}\left(1+\left(2 a q_{1}\right)^{2 / 3}\right) \times \\
\left(1-e^{2}-A_{1}-A_{2}+A_{1}\left(a q_{1}\right)^{-2 / 3}-2\left(a q_{2}\right)^{2 / 3} \times\right. \\
\left(1-e^{2}-A_{1}-A_{2}+A_{2}\left(a q_{2}\right)^{-2 / 3}\right)
\end{array}\right]^{1 / 2}
\end{aligned}
$$

\section{Stability of the triangular equilibrium points}

In order to study the stability of the triangular points, the locations are denoted by $\left(\xi_{0}, \eta_{0}\right)$. Let there be a small displacement in $\left(\xi_{0}, \eta_{0}\right)$ which is given by $(\sigma, \beta)$. Considering only the linear terms in $\sigma, \beta$ the variation equations of motions defined by equation (2.1) take the form:

$$
\begin{gathered}
\sigma^{\prime \prime}-2 \beta^{\prime}=\sigma \Omega_{\xi \xi}^{* 0}+\beta \Omega_{\xi \eta}^{* 0}, \\
\beta^{\prime \prime}+2 \sigma^{\prime}=\sigma \Omega_{\xi \eta}^{* 0}+\beta \Omega_{\eta \eta}^{* 0} .
\end{gathered}
$$

The second partial derivatives of $\Omega$ are denoted by subscripts .The superscripts 0 denotes the partial derivatives at the equilibrium points $\left(\xi_{0}, \eta_{0}\right)$

The corresponding characteristics equation of the system (3.1) can be obtained as the biquadratic equation:

$$
\lambda^{4}+\left(4-C_{\xi \xi}-C_{\eta \eta}\right) \lambda^{2}+\left(C_{\xi \xi} C_{\eta \eta}-C_{\xi \eta}^{2}\right)=0 .
$$

Where,

$C_{\xi \xi}=\left(\Omega_{\xi \xi}{ }^{0}\right)$,

$C_{\xi \eta}=\left(\Omega_{\xi \eta}{ }^{0}\right)$,

$C_{\eta \eta}=\left(\Omega_{\eta \eta}{ }^{0}\right)$

Assuming the solution of the system of equation (3.1) to be of the form:

$u=A \exp (\lambda f), v=B \exp (\lambda f)$

Substituting into equation (3.1), we obtain:

$$
\begin{aligned}
& \left(\lambda^{2}-C_{\xi \xi}\right) A-\left(2 \lambda+C_{\xi \eta}\right) B=0, \\
& \left(\lambda^{2}-C_{\eta \eta}\right) B+\left(2 \lambda-C_{\xi \eta}\right) A=0,
\end{aligned}
$$

Assuming ,

$$
a=1-\alpha, q_{1}=1-\chi_{1}, q_{2}=1-\chi_{2},
$$

We obtain the characteristics equation as: 


$$
\lambda^{4}+\left(4-3 \varphi_{1}\right) \lambda^{2}+\frac{27}{4} \mu(1-\mu)+\varphi_{2}=0
$$

Where,

$$
\begin{aligned}
& \left(4-C_{\xi \xi}-C_{\eta \eta}\right)=4-3 \varphi_{1}, \\
& \left(C_{\xi \xi} C_{\eta \eta}-C_{\xi \eta}^{2}\right)=\frac{27}{4} \mu(1-\mu)+\varphi_{2}, \\
& \varphi_{1}=\left(1-e^{2}\right)^{-1 / 2} \times\left[\begin{array}{l}
\left.1+(1-\mu) A_{1}\left(1+\frac{2}{3} \alpha+\frac{2}{3} \chi_{1}\right)\right] . \\
+\mu A_{2}\left(1+\frac{2}{3} \alpha+\frac{2}{3} \chi_{2}\right)
\end{array}\right] \\
& \varphi_{1}=\left(1-e^{2}\right)^{-1 / 2}\left(1+(1-\mu) A_{1}+\mu A_{2}\right) . \\
& \varphi_{2}=3 \mu(1-\mu) \alpha+\frac{3 \mu(1-\mu)}{2}\left(\chi_{1}+\chi_{2}\right)+ \\
& 9 \mu(1-\mu)\left(A_{1}+A_{2}\right)+\frac{45}{4} \mu(1-\mu) e^{2} . \\
& \varphi_{2}=3 \mu(1-\mu)\left[\alpha+\frac{1}{2}\left(\chi_{1}+\chi_{2}\right)+3\left(A_{1}+A_{2}\right)+\frac{15}{4} e^{2}\right] .
\end{aligned}
$$

Where only linear terms in $e^{2}, A_{1}, A_{2}, \alpha, \chi_{1}, \chi_{2}$ are taken into consideration

The chacteristics roots are obtained as:

$$
\lambda_{1,2}^{2}=\frac{-\left(4-3 \varphi_{1}\right) \pm\left[\begin{array}{l}
\left(4-3 \varphi_{1}\right)^{2}- \\
\left.27 \mu(1-\mu)-4 \varphi_{2}\right)
\end{array}\right]^{1 / 2}}{2} .
$$

The critical value is defined as:

$$
\begin{aligned}
\mu_{c}= & \frac{1}{2}\left(\left(1-\sqrt{\frac{23}{7}}\right)\right)-\frac{1}{9}\left(1+\frac{13}{\sqrt{69}}\right) A_{1}+ \\
& \frac{1}{9}\left(1-\frac{13}{\sqrt{69}}\right) A_{2}-\frac{2}{27 \sqrt{69}}\left(\chi_{1}+\chi_{2}\right) \\
& -\frac{4}{27 \sqrt{69}} \alpha-\frac{14}{9 \sqrt{69}} e^{2} .
\end{aligned}
$$

Considering the perturbed motion of $\mathrm{L}_{4}$ around different binary systems with the following initial conditions as:

$$
\begin{aligned}
& u_{0}=v_{0}=10^{-5}, f=0, \\
& \alpha=0.1, A_{1}=0.01, A_{2}=0.02 .
\end{aligned}
$$

Now, for the binary system, Achird, equation (3.3) gives:

$$
\mathrm{C}_{11}=1.8314, \mathrm{C}_{22}=0.7972, \mathrm{C}_{12}=0.3044 \text {. }
$$

The roots of the characteristics equations are:

$$
\begin{aligned}
& \lambda_{1,2}= \pm(0.7699+0.9165 i), \\
& \lambda_{3,4}= \pm(0.7699-0.9165 i) .
\end{aligned}
$$

The solution of the perturbed motion around $\mathrm{L}_{4}$ may be written as: $u(f)=\sum_{j=1}^{4} A_{j} e^{\lambda j f}, v(f)=\sum_{j=1}^{4} B_{j} e^{\lambda_{j} f}$

The constants $A_{j}$ and $B_{j}$ are not independent; they are related by the equation (3.5). The relation can be written as:

$$
B_{j}=\frac{\lambda_{j}^{2}-C_{11}}{2 \lambda_{j}+C_{12}} A_{j}
$$

$j=1,2,3,4$.

Applying the initial conditions to the solution, the solution may be expressed as:

$$
\begin{aligned}
u(f)= & 0.4010 \cos (0.9165 f) \\
& +0.2722 \sin (0.9165 f) \\
& -0.3950 e^{0.7699 f} \\
+ & 0.006 e^{-0.7699 f}, \\
v(f)= & 0.3776 \cos (0.9165 f) \\
& -0.41702 \sin (0.9165 f) \\
& +0.0078 e^{0.7699 f} \\
& +0.3776 e^{-0.7699 f} .
\end{aligned}
$$

Similarly for the binary system Luyten; we have:

$\mathrm{C}_{11}=1.8631, \mathrm{C}_{22}=0.5096, \mathrm{C}_{12}=-0.1148$.

The roots of the characteristics equation is:

$$
\begin{aligned}
\lambda_{1,2}= \pm & (0.8715+0.9050 i), \\
\lambda_{3,4}= \pm & (0.8715-0.9050 i) . \\
u(f)= & 0.0714 \cos (0.9050 f) \\
& +2.0144 \sin (0.9050 f) \\
& -0.089 e^{0.8715 f} \\
& -0.006 e^{-0.8715 f}, \\
v(f)= & -0.213 \cos (0.9050 f) \\
& -3.9466 \sin (0.9050 f) \\
& -0.213 e^{0.8715 f} \\
& +0.0928 e^{-0.8715 f} .
\end{aligned}
$$

For the binary system $\alpha$ Cen $\mathrm{AB}$, we have

$\mathrm{C}_{11}=1.8610, \mathrm{C}_{22}=0.7404, \mathrm{C}_{12}=0.1961$

The roots of the characteristics equation is:

$$
\lambda_{1,2}= \pm(0.7937+0.91225 i)
$$

$\lambda_{3,4}= \pm(0.7937-0.91225 i)$ 
Similarly,

$$
\begin{aligned}
u(f)= & -0.2602 e^{0.7937 f} \\
& -0.1586 e^{-0.7937 f} \\
- & 0.1016 \cos (0.91225 f) \\
& +3.722 \sin (0.91225 f), \\
v(f)= & -0.1888 e^{0.7937 f} \\
& +0.2842 e^{-0.7937 f} \\
& +0.0954 \cos (0.91225 f) \\
& -4.245 \sin (0.91225 f) .
\end{aligned}
$$

For the binary system Kruger-60, we have $\mathrm{C}_{11}=1.8644, \mathrm{C}_{22}=0.8986, \mathrm{C}_{12}=0.2844$.

The roots of the characteristics equation is:

$$
\lambda_{1,2}= \pm(0.7215+0.9236 i) \text {, }
$$$$
\lambda_{3,4}= \pm(0.7215-0.9236 i) \text {, }
$$

$$
\begin{aligned}
u(f)= & 0.4168 e^{0.7215 f} \\
& +0.035 e^{-0.7215 f} \\
& -0.3818 \cos (0.9236 f) \\
& -4.3454 \sin (0.9236 f),
\end{aligned}
$$

$$
\begin{aligned}
v(f)= & -0.0252 e^{0.7215 f} \\
& +0.4038 e^{-0.7215 f} \\
& -0.3768 \cos (0.9236 f) \\
& +3.6236 \sin (0.9236 f) .
\end{aligned}
$$

For the binary system Xi-Bootis, we have

$\mathrm{C}_{11}=1.8610, \mathrm{C}_{22}=0.7404, \mathrm{C}_{12}=0.1961$.

The roots of the characteristics equation is:

$$
\begin{aligned}
& \lambda_{1,2}= \pm(0.7850+0.9195 i), \\
& \lambda_{3,4}= \pm(0.7850-0.9195 i),
\end{aligned}
$$

$$
\begin{aligned}
u(f)= & -0.0824 e^{0.7850 f} \\
& +0.3190 e^{-0.7850 f} \\
& +0.1183 \cos (0.9195 f) \\
& -4.1788 \sin (0.9195 f), \\
v(f)= & -0.3214 e^{0.7850 f} \\
& +0.0672 e^{-0.7850 f} \\
& -0.2542 \cos (0.9195 f) \\
& +0.1756 \sin (0.9195 f) .
\end{aligned}
$$

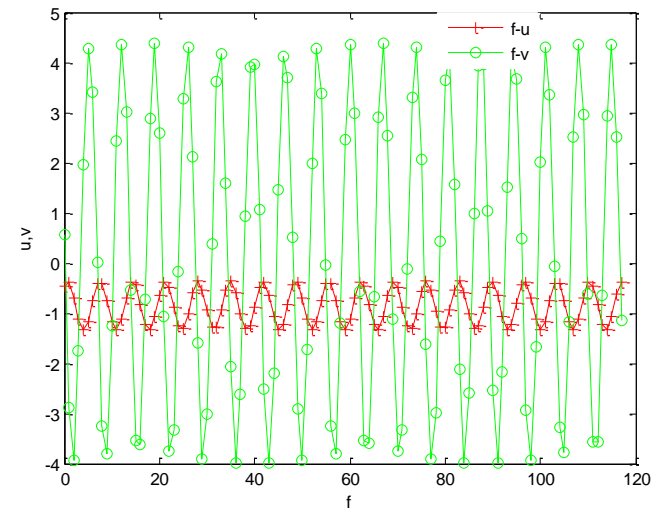

Fig. 1: The Graph of the Function $f-u$ and $f-v$ for $f \epsilon[0,37.75 \pi]$ in case of Achird.

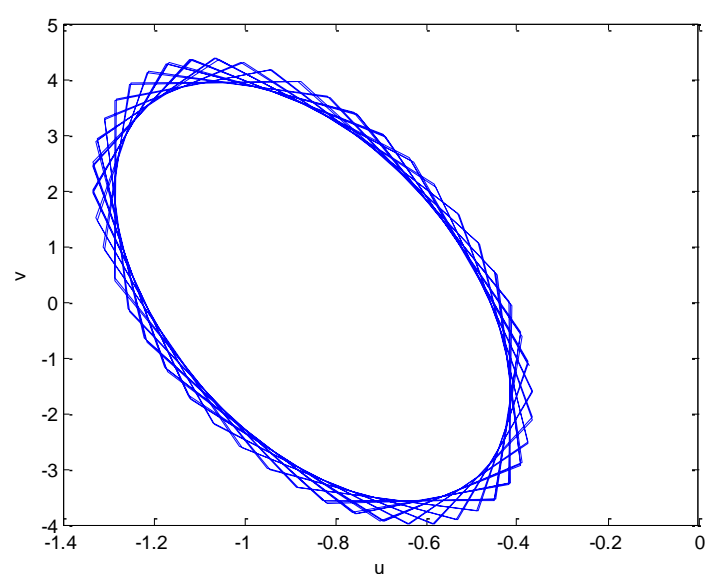

Fig. 2: The u-v Graph for Achird in range $f \in[0,37.75 \pi]$.

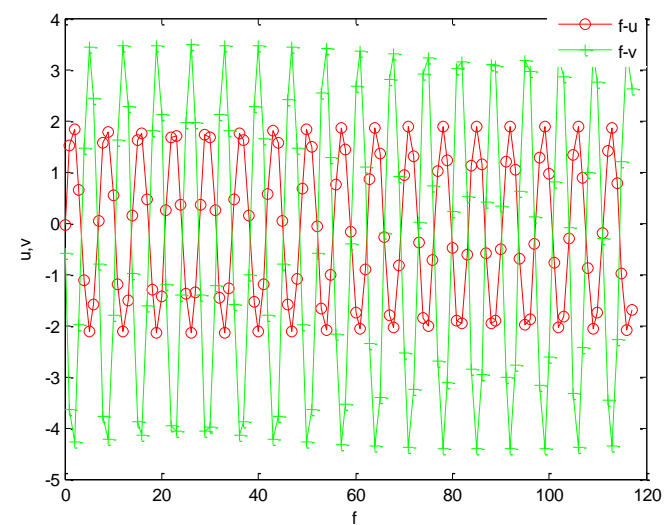

Fig. 3: The Graph of the Function $f-u$ and $f-v$ for $f \in[0,37.75 \pi]$ in case of Luyten.

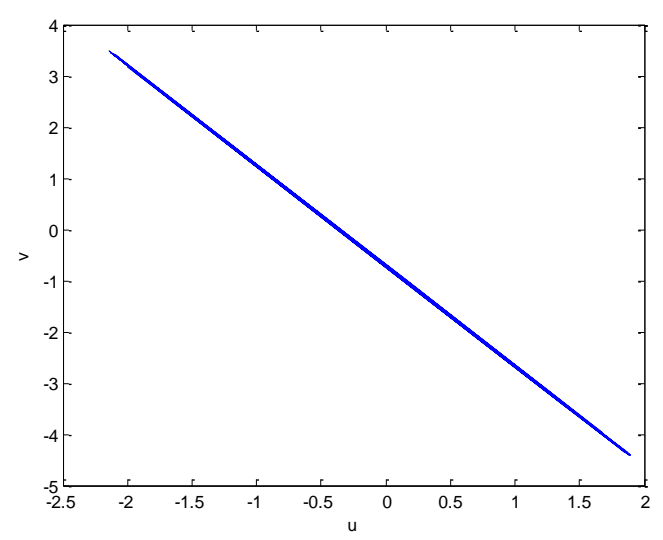

Fig. 4: The u-v Graph for Luyten in range $f \in[0,37.75 \pi]$. 


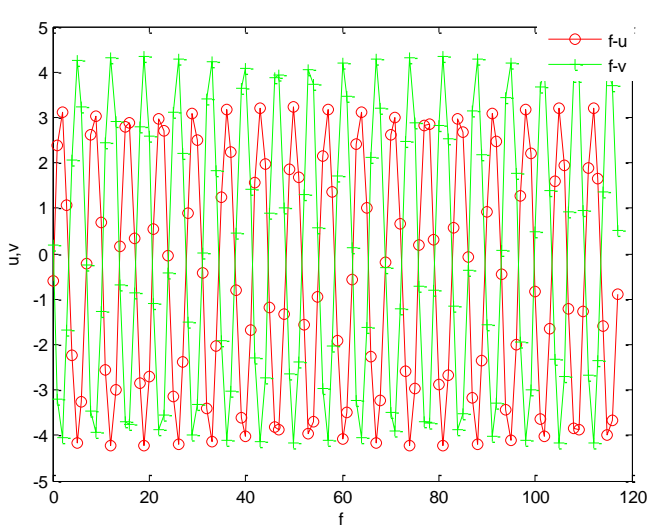

Fig. 5: The Graph of the Function $f-u$ and $f-v$ for $f \in[0,37.75 \pi]$ in case of $\alpha$ Cen $\mathrm{AB}$.

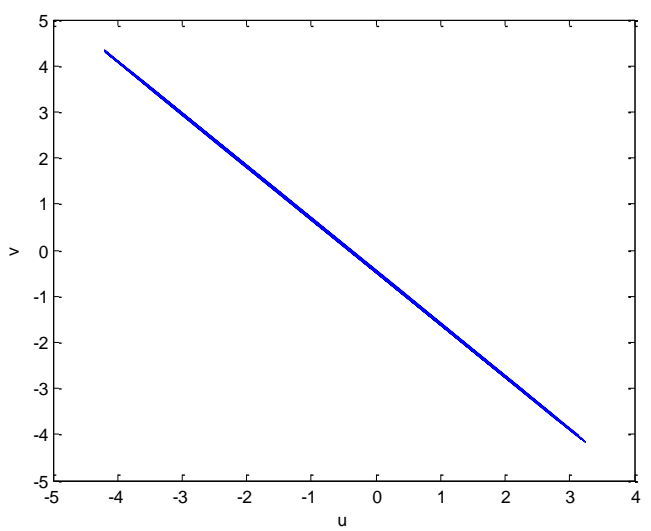

Fig. 6: The u-v Graph for $\alpha$ Cen AB. in range $f \in[0,37.75 \pi]$.

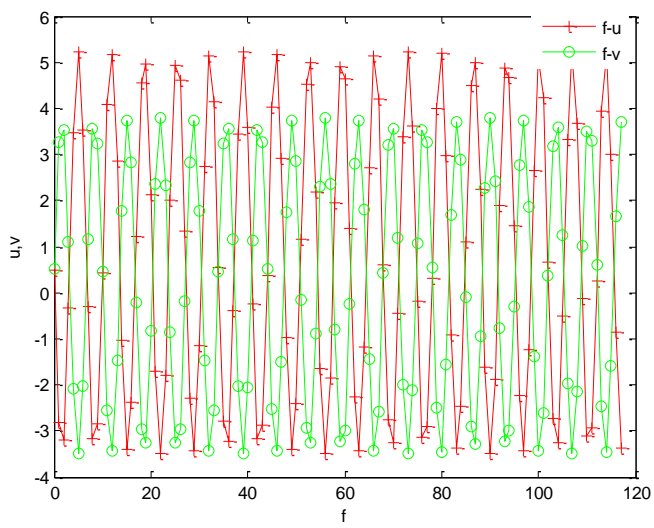

Fig. 7: The Graph of the Function $f-u$ and $f-v$ for $f \in[0,37.75 \pi]$ in Case of Kruger- 60

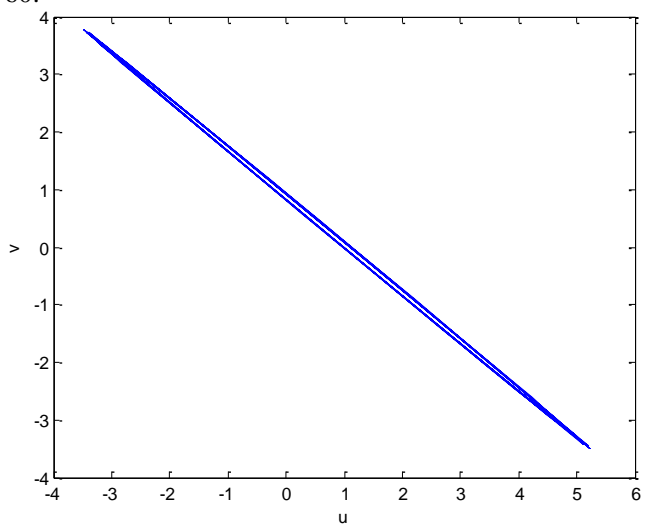

Fig. 8: The u-v Graph for Kruger-60 in range $f \in[0,37.75 \pi]$.

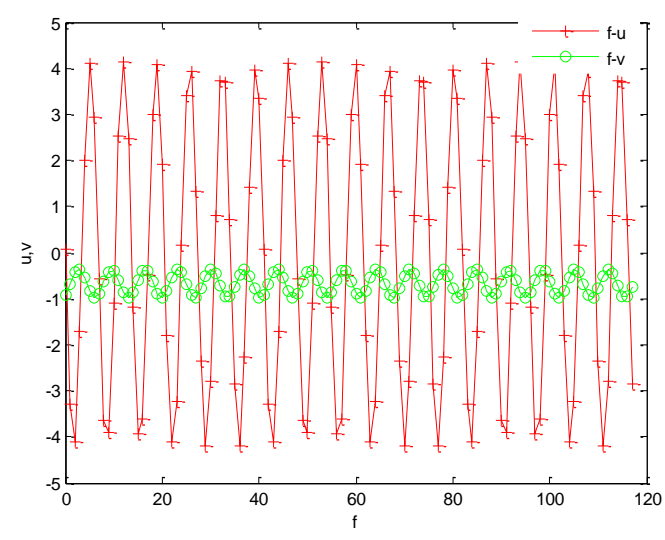

Fig. 9: The Graph of the Function $f-u$ and $f-v$ for $f \in[0,37.75 \pi]$ in case of Xi- Bootis.

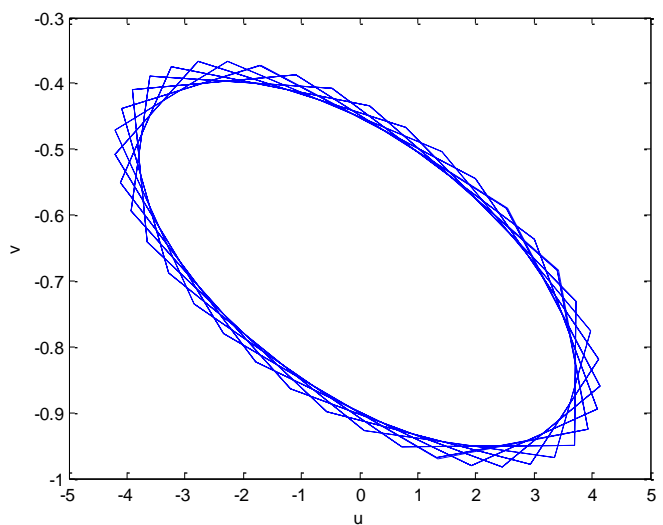

Fig. 10: The u-v Graph for Xi-Bootis in range $f \in[0,37.75 \pi]$.

The simulations of the solutions $u(f), v(f)$ and $u-v$ for binary systems are shown in Figs.1-10 for $\mathrm{f} \in[0,37.75 \pi]$. The solutions of the perturbed motion around L4 is given by $u(f)$ and $v(f)$ function for the binary systems. The graph of the function $u(f)$ and $v$ (f) is plotted in the vicinity of L4 in Figs. 1, 3, 5, 7, and 9. It is clear from the Figs.1, 3, 5, 7, 9 that the motion of the infinitesimal mass at the triangular points is oscillatory. $u(f)-v(f)$ curve is plotted in Figs 2, 4, 6, 8, 10 in the range $f \in[0,37.75 \pi]$.

\section{Trajectories of the infinitesimal mass around the triangular equilibrium points}

The trajectory of the infinitesimal mass around $\mathrm{L}_{4}$ of the triangular equilibrium points for the binary systems in the ERTBP considering both the primaries as radiating and oblate has been discussed. In order to find the trajectory, the three equations of (2.1) are multiplied by $\xi^{\prime}, \eta^{\prime}, \zeta^{\prime}$ in turns and added, which finally gives:

$\xi^{\prime} \xi^{\prime \prime}+\eta^{\prime} \eta^{\prime \prime}+\zeta^{\prime} \zeta^{\prime \prime}=\frac{\partial \Omega}{\partial \xi} \xi^{\prime}+\frac{\partial \Omega}{\partial \eta} \eta^{\prime}+\frac{\partial \Omega}{\partial \zeta} \zeta^{\prime}$

Since $\Omega$ does not contain time explicitly, therefore equation (4.1) can be integrated and given as follows:

$\xi^{2}+\eta^{2}+\zeta^{\prime 2}=\int \frac{d \Omega}{\sqrt{1-e^{2}}}+C$.

Which, on further simplification gives: 
$\xi^{2}+\eta^{2}+\zeta^{2}-\frac{1}{\sqrt{1-e^{2}}}\left(\xi^{2}+\eta^{2}+\zeta^{2}\right)-$

$\frac{2}{\sqrt{1-e^{2}}} \frac{1}{n^{2}} \times$

$\left(\frac{(1-\mu) q_{1}}{r_{1}}+\frac{\mu q_{2}}{r_{2}}+\frac{(1-\mu) A_{1} q_{1}}{2 r_{1}^{3}}+\frac{\mu A_{2} q_{2}}{2 r_{2}^{3}}\right)=C$.

Therefore in planar $(\zeta=0)$ ERTBP the trajectory of the infinitesimal are obtained from the equation (4.3) as:

$$
\begin{aligned}
& \left(\xi^{2}+\eta^{2}\right)-\frac{2}{n^{2}} \times \\
& \left(\frac{(1-\mu) q_{1}}{r_{1}}+\frac{\mu q_{2}}{r_{2}}+\frac{(1-\mu) A_{1} q_{1}}{2 r_{1}^{3}}+\frac{\mu A_{2} q_{2}}{2 r_{2}^{3}}\right)=C *
\end{aligned}
$$

Where

$$
C^{*}=C \sqrt{1-e^{2}}
$$

Geometrically, this means that at every time (or any value of true anomaly, f ) different sets of trajectory can be constructed for different values of radiation pressure and oblateness parameter.

The trajectory of the infinitesimal mass is traced with the help of equation (4.4) using Matlab (7.1) around the triangular equilibrium points, for the binary systems. (Achird, Luyten, Alpha Cen AB, Kruger-60 and Xi-Bootis) by varying the oblateness parameter.

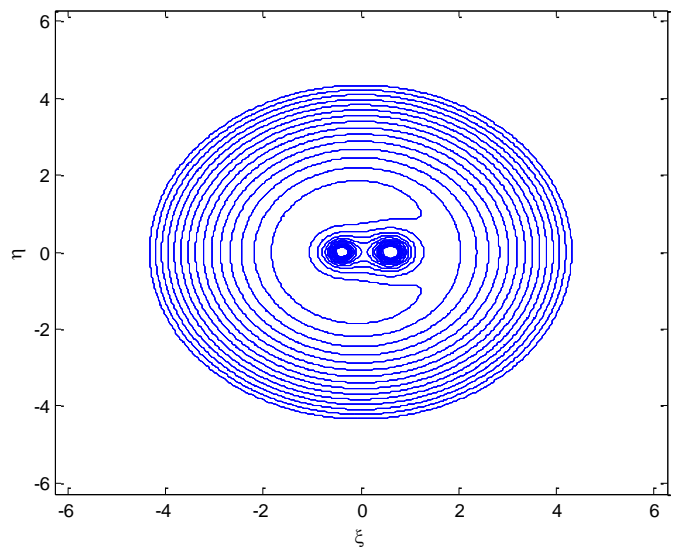

Fig. 11: Trajectory for Achird when $A_{1}=0.001, A_{2}=0.002$.

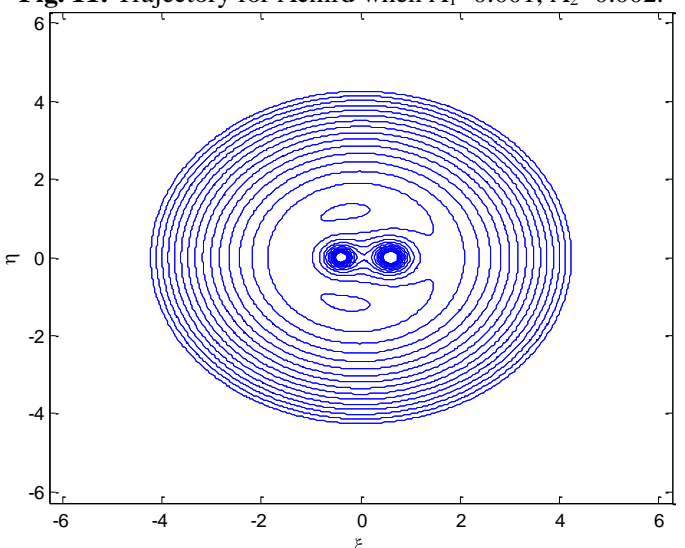

Fig. 12: Trajectory for Achird when $A_{1}=0.01, A_{2}=0.02$.

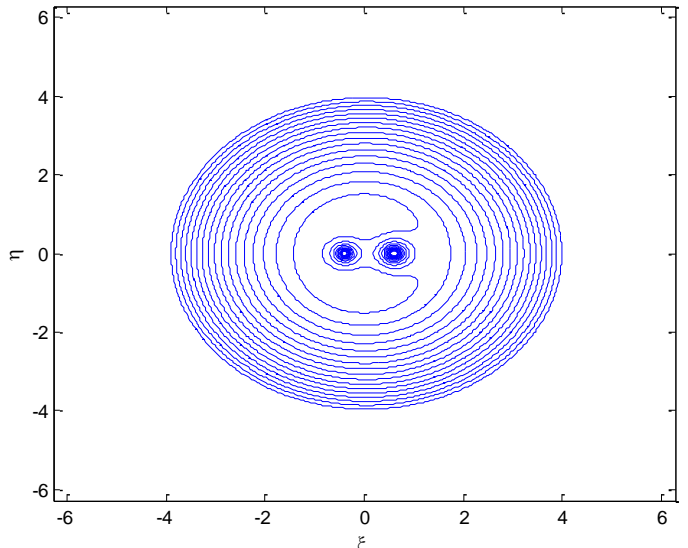

Fig. 13: Trajectory for Achird At $A_{1}=0.1, A_{2}=0.2$.

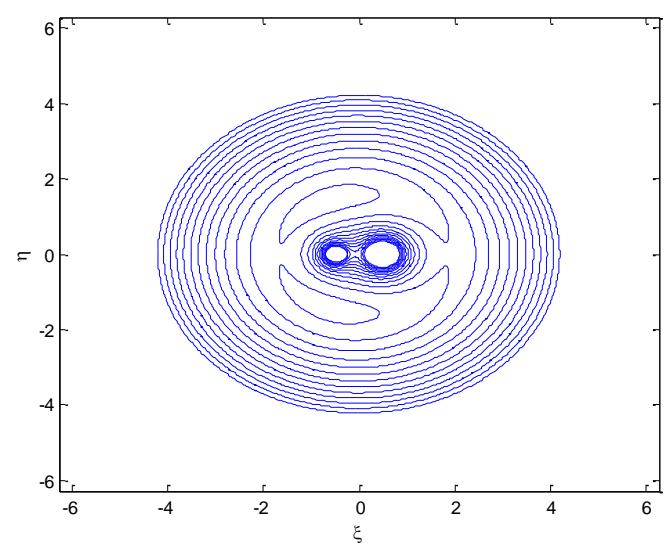

Fig. 14: Trajectory for Luyten when $A_{1}=0.001, A_{2}=0.002$.

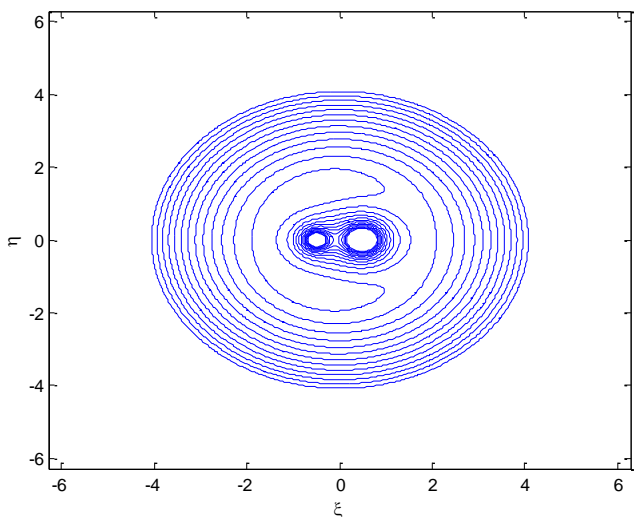

Fig. 15: Trajectory for Luyten when $A_{1}=0.01, A_{2}=0.02$.

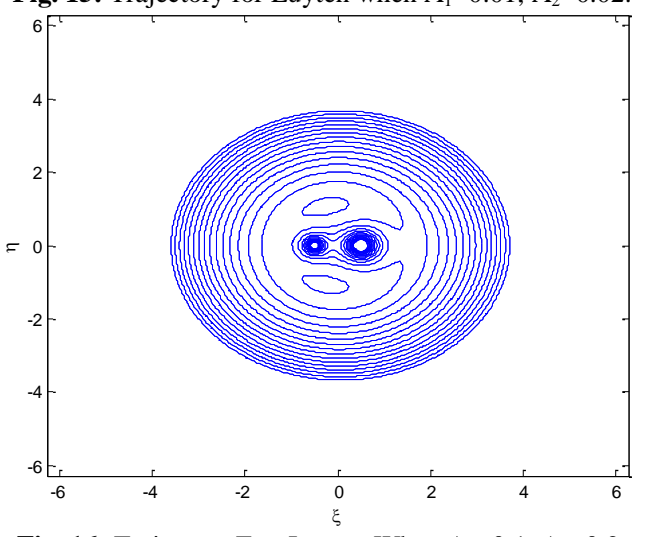

Fig. 16: Trajectory For Luyten When $A_{1}=0.1, A_{2}=0.2$. 


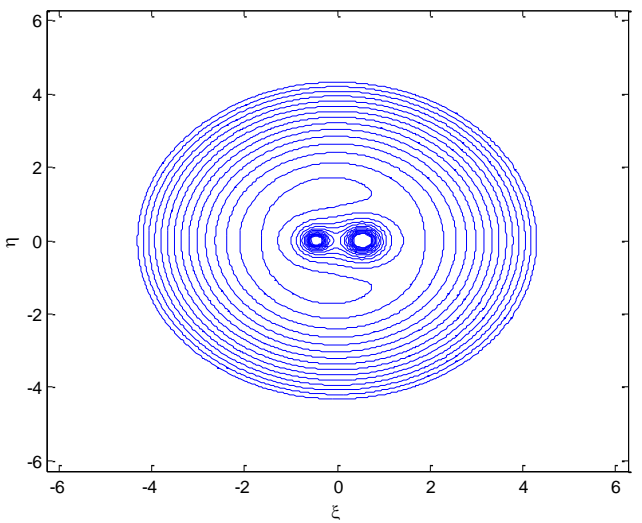

Fig. 17: Trajectory for $\alpha$ Cen $A B$ when $A_{1}=0.001, A_{2}=0.002$.

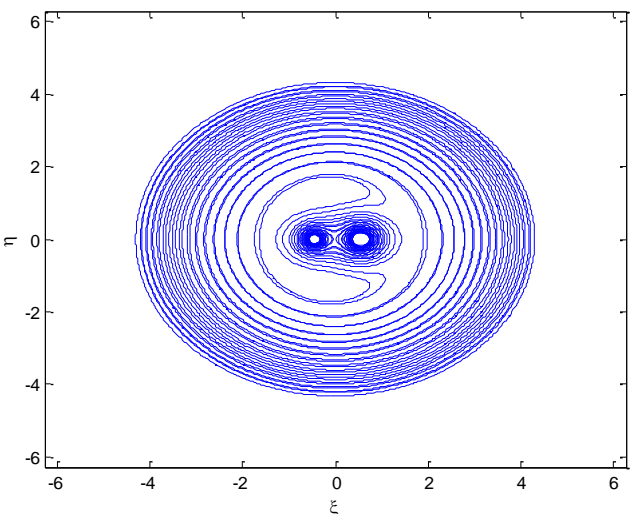

Fig. 18: Trajectory for $\alpha$ Cen $\mathrm{AB}$ when $\mathrm{A}_{1}=0.01, \mathrm{~A}_{2}=0.02$.

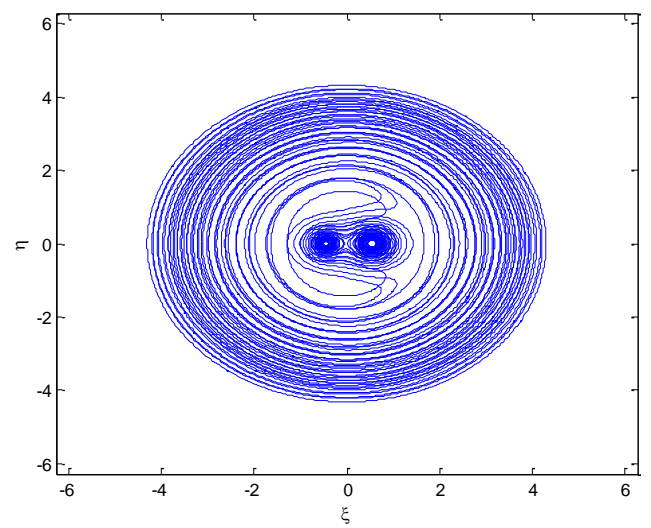

Fig. 19: Trajectory for $\alpha$ Cen $A B$ when $A_{1}=0.01, A_{2}=0.02$.

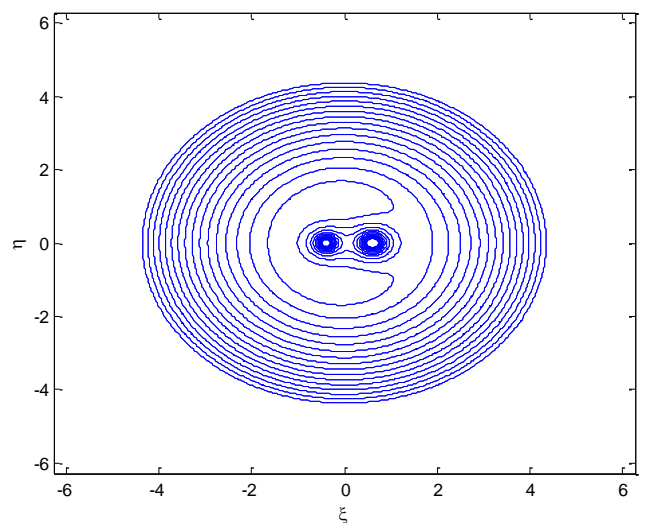

Fig. 20: Trajectory for Kruger- 60 when $A_{1}=0.001, A_{2}=0.002$.

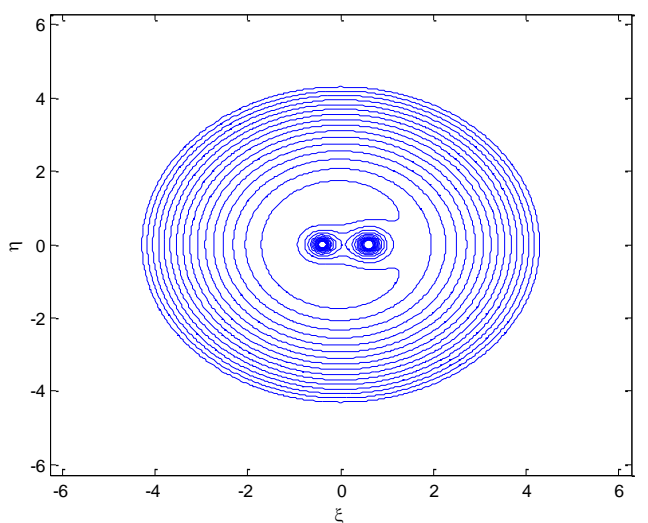

Fig. 21: Trajectory for Kruger-60 when $A_{1}=0.01, A_{2}=0.02$.

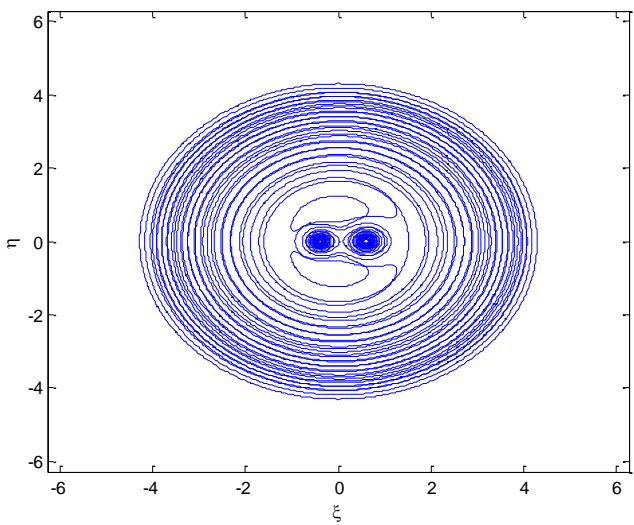

Fig. 22: Trajectory for Kruger- 60 when $A_{1}=0.1, A_{2}=0.2$

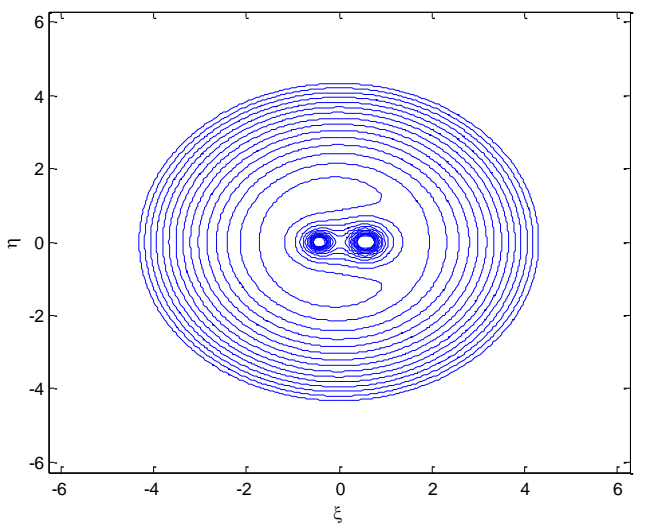

Fig. 23: Trajectory for Xi-Bootis when $A_{1}=0.001, A_{2}=0.002$.

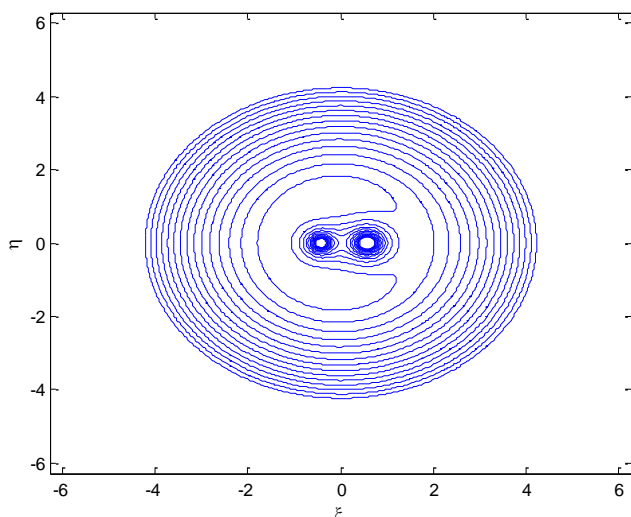

Fig. 24: Trajectory for Xi-Bootis when $A_{1}=0.01, A_{2}=0.02$. 


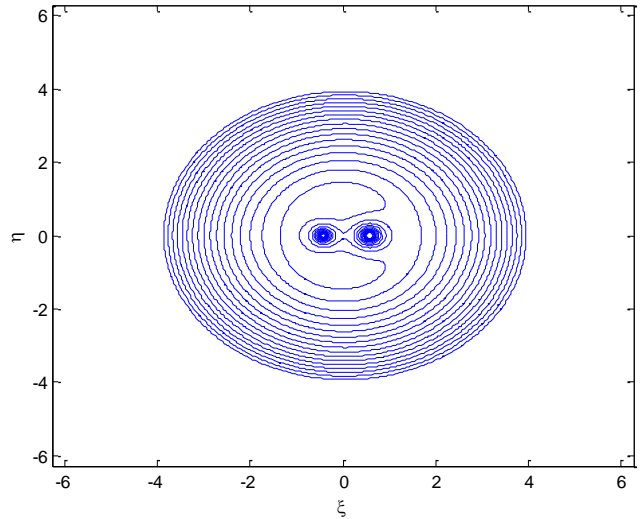

Fig. 25: Trajectory for Xi-Bootis when $A_{1}=0.1, A_{2}=0.2$.

The trajectory for Achird is represented by Fig.11, Fig.12, Fig.13; likewise Fig.14, Fig.15, Fig.16 represent the same for binary system Luyten. The same is represented by Fig.17, Fig.18, Fig.19 for binary system $\alpha$ Cen AB.Similarly Fig.20, Fig. 21, Fig. 22 represent trajectory for the binary system Kruger-60 and Fig 23, Fig 24, Fig 25 for binary system Xi-Bootis. It can be seen that due to change in oblateness there is significant change in two dimensional trajectories for the binary systems. The three dimensional trajectory of the infinitesimal mass around the triangular points for the binary systems is represented by Figs.2640 for different values of oblateness parameter. It is found that the three dimensional figure exhibits almost the same nature and behavior which is obvious from Figs.26-40.

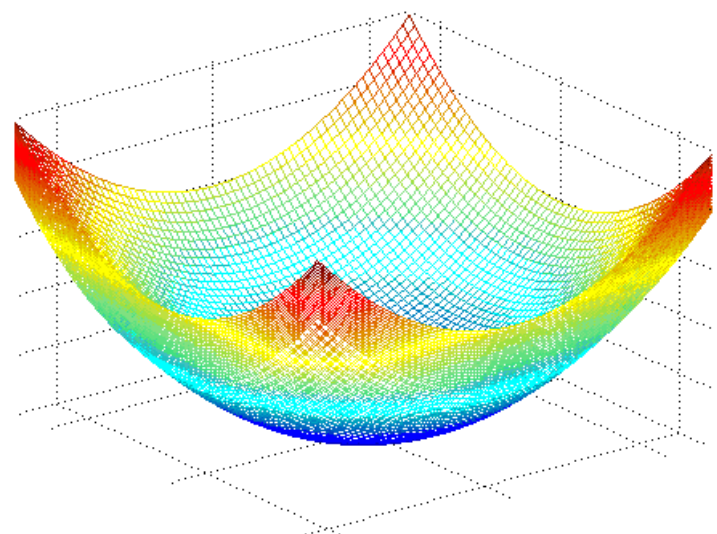

Fig. 26: Three Dimensional Trajectory for Achird when $A_{1}=0.001$, $\mathrm{A}_{2}=0.002$.

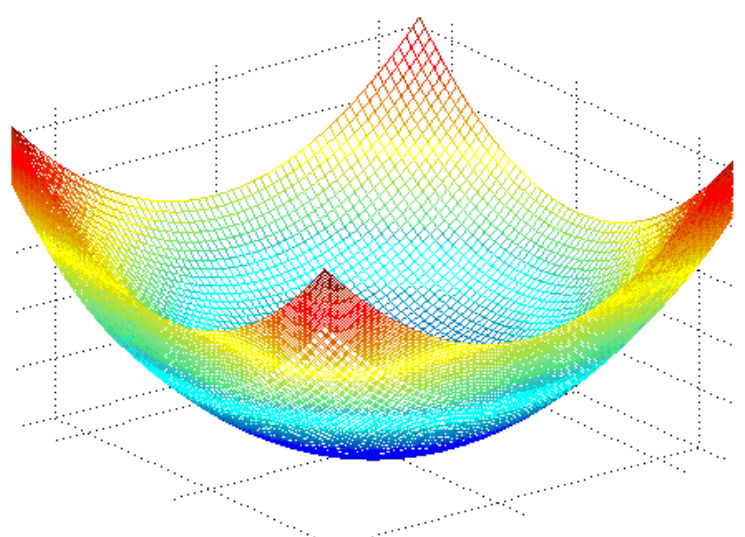

Fig. 27: Three Dimensional Trajectory for Achird when $A_{1}=0.01$, $\mathrm{A}_{2}=0.02$.

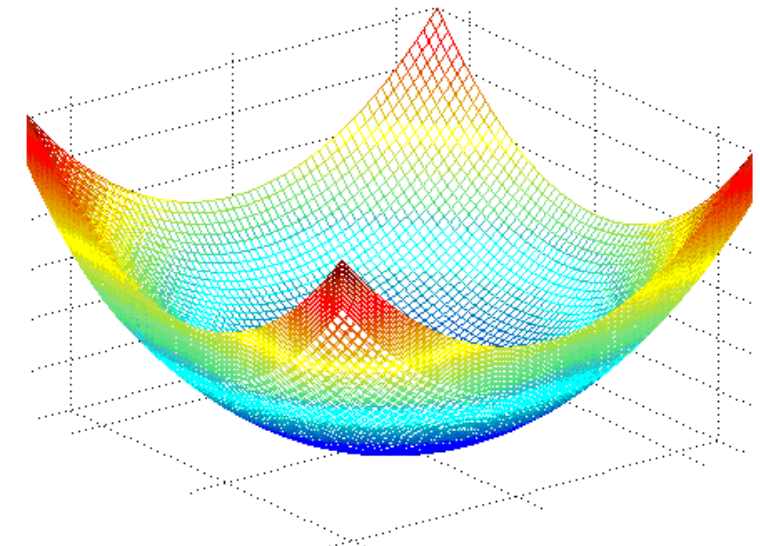

Fig. 28: Three Dimensional Trajectory for Achird when $A_{1}=0.1, A_{2}=0.2$.

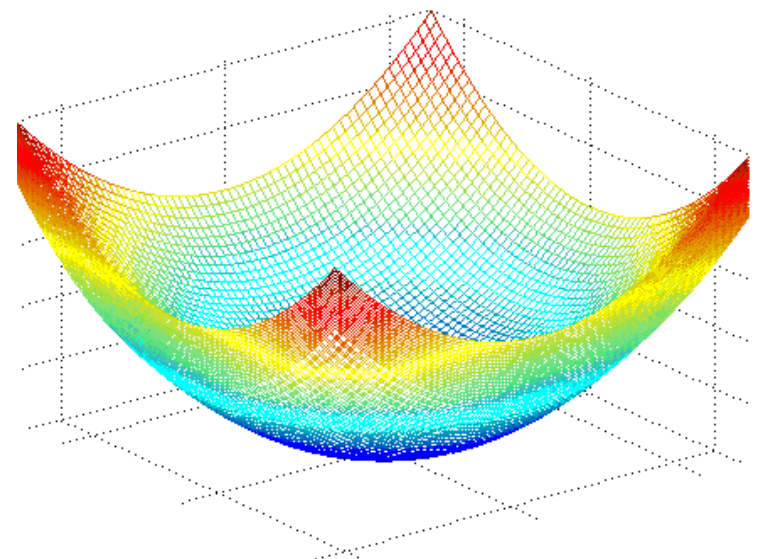

Fig. 29: Three Dimensional Trajectory for Luyten when $A_{1}=0.001$, $\mathrm{A}_{2}=0.002$.

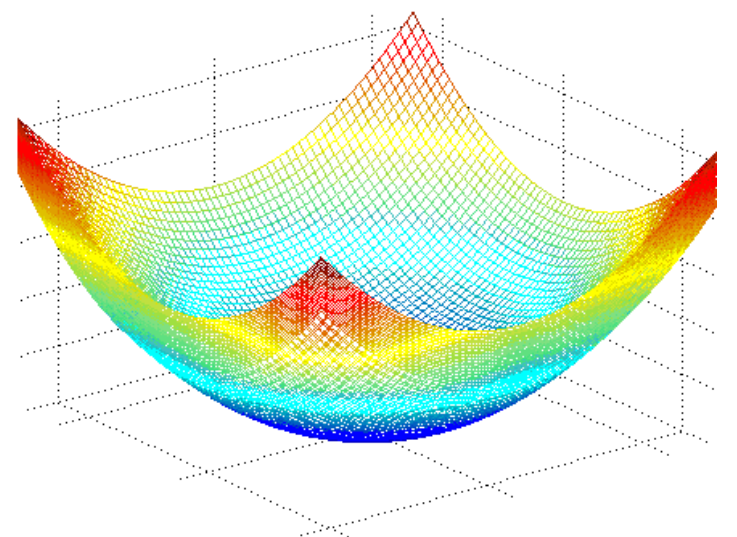

Fig. 30: Three Dimensional Trajectory for Luyten when $A_{1}=0.01$, $\mathrm{A}_{2}=0.02$.

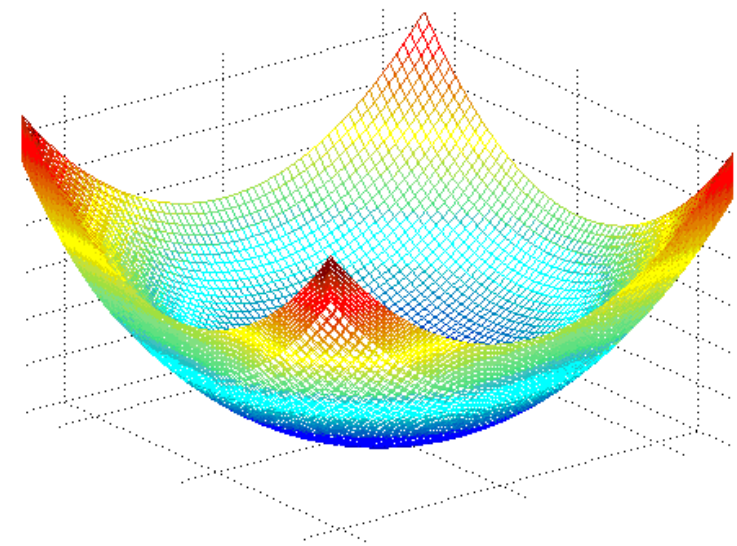

Fig. 31: Three Dimensional Trajectory for Luyten when $A_{1}=0.1, A_{2}=0.2$. 


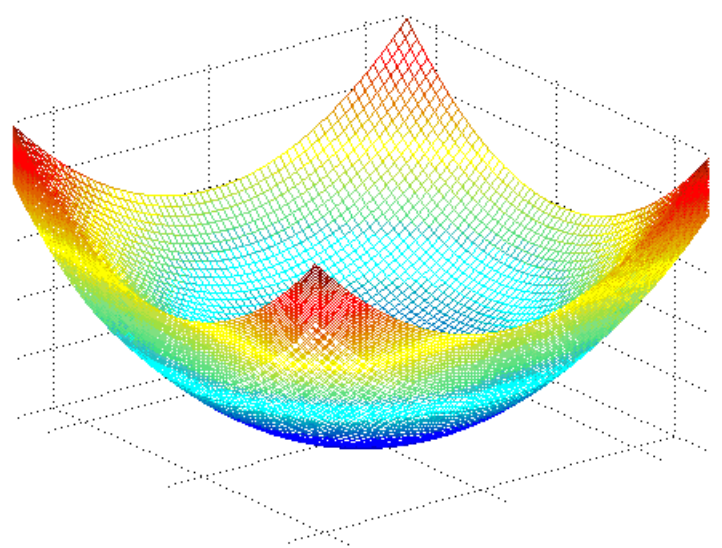

Fig. 32: Three Dimensional Trajectory for $\alpha$ Cen $A B$ when $A_{1}=0.001$, $\mathrm{A}_{2}=0.002$.

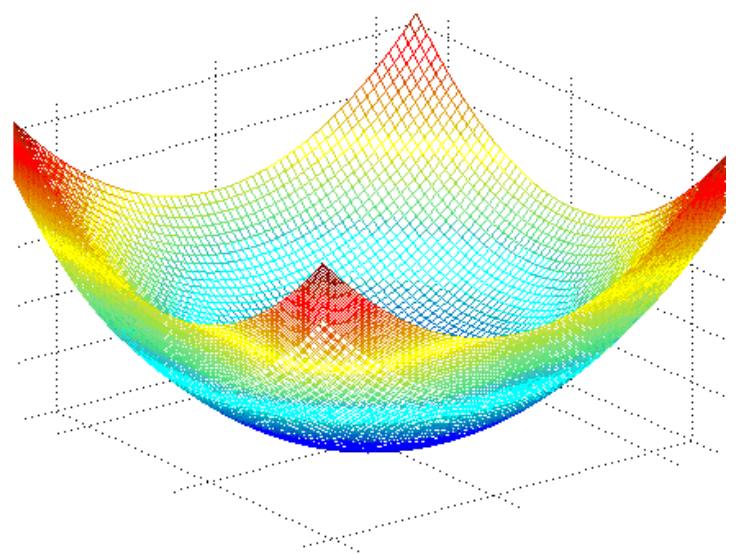

Fig. 33: Three Dimensional Trajectory for $\alpha$ Cen $A B$ when $A_{1}=0.01$, $\mathrm{A}_{2}=0.02$.

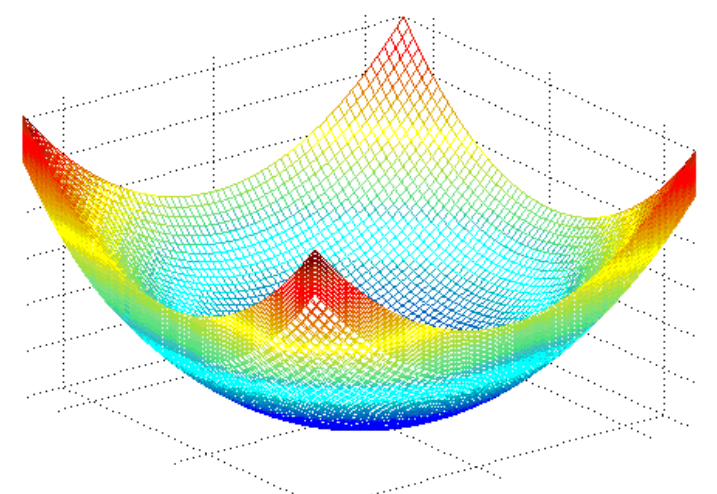

Fig. 34: Three Dimensional Trajectory for $\alpha$ Cen $A B$ when $A_{1}=0.1$, $\mathrm{A}_{2}=0.2$.

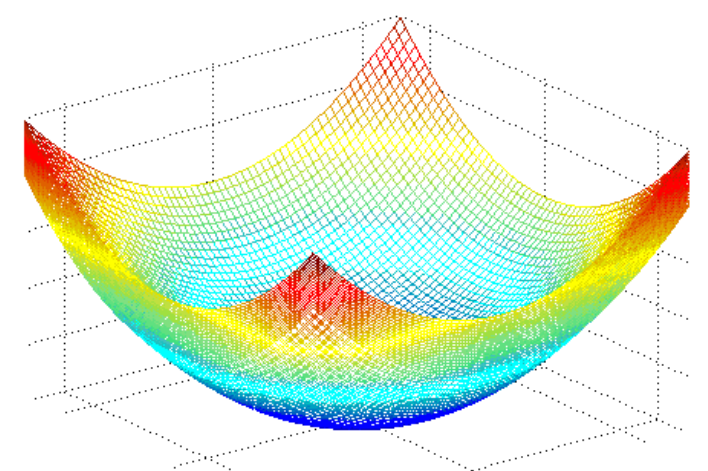

Fig. 35: Three Dimensional Trajectory for Kruger- 60 when $A_{1}=0.001$, $\mathrm{A}_{2}=0.002$.

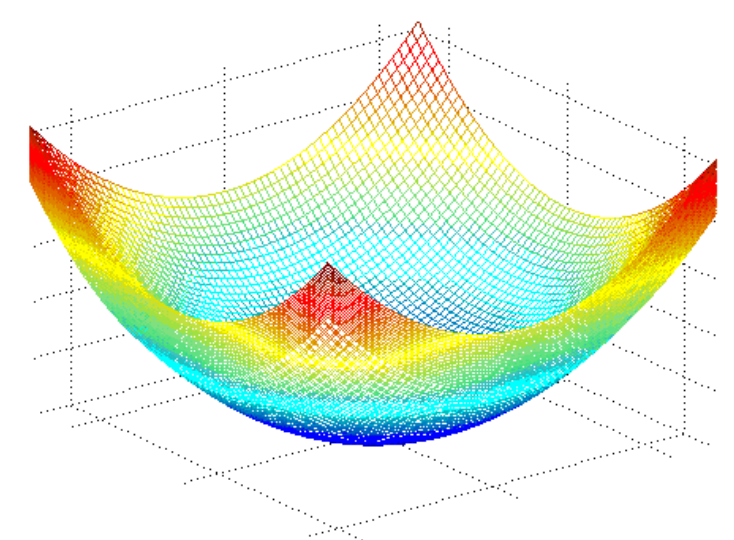

Fig. 36: Three Dimensional Trajectory for Kruger-60 when $A_{1}=0.01$, $\mathrm{A}_{2}=0.02$.

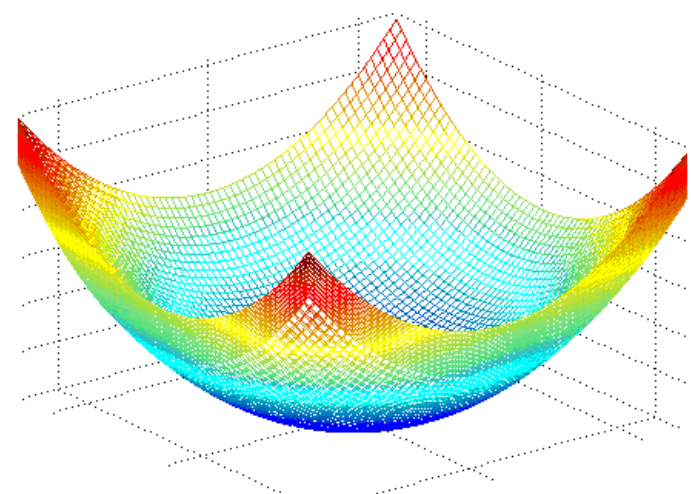

Fig. 37: Three Dimensional Trajectory for Kruger-60 when $A_{1}=0.1$, $\mathrm{A}_{2}=0.2$.

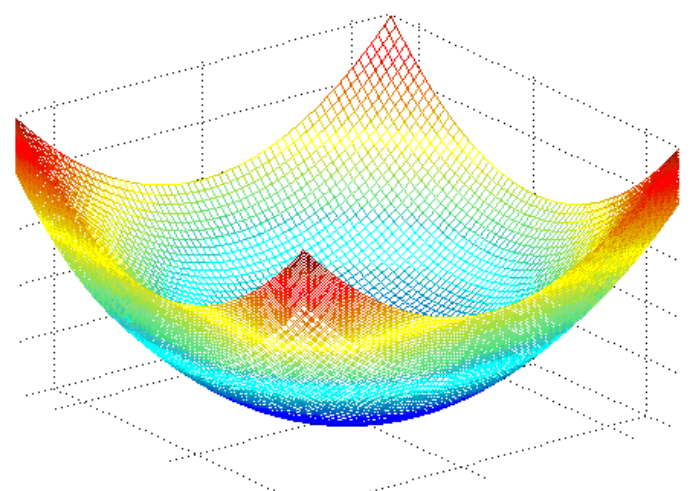

Fig. 38: Three Dimensional Trajectory for Xi- Bootis when $A_{1}=0.001$, $\mathrm{A}_{2}=0.002$.

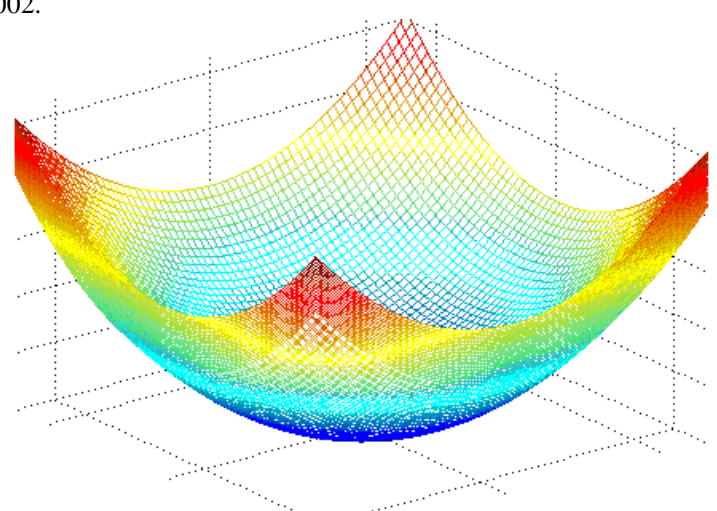

Fig. 39: Three Dimensional Trajectory for Xi-Bootis when $A_{1}=0.01$, $\mathrm{A}_{2}=0.02$. 


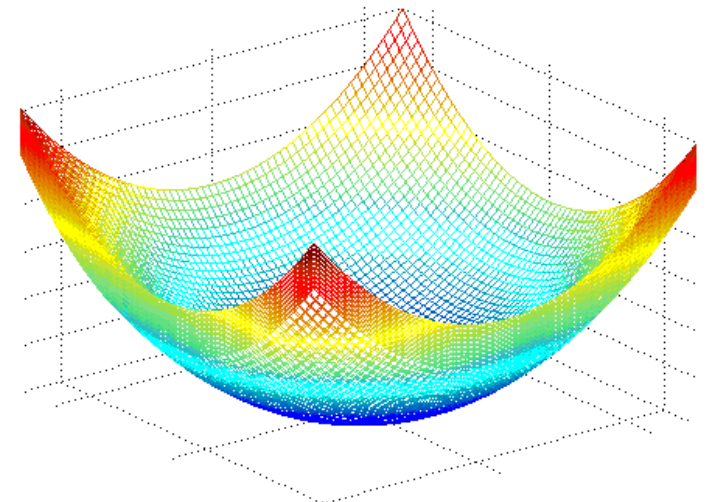

Fig. 40: Three Dimensional Trajectory for Xi-Bootis when $A_{1}=0.1$, $\mathrm{A}_{2}=0.2$.

\section{Discussion and Conclusion}

The trajectory of the infinitesimal mass around $\mathrm{L}_{4}$ of the triangular equilibrium points are studied in the framework of ERTBP taking both the primaries as radiating and oblate spheroid. The study is done for the binary systems (Achird, Luyten, Alpha Cen AB, Kruger-60 and Xi-Bootis)

The solutions are obtained by plotting u (f), v (f) graph for the binary systems. Singh and Umar (2012b) studied the locations of the triangular points for the binary systems in particular and found that there is shift in both the coordinates due to change in oblatenes parameter. They also studied the region of stability by varying radiation pressure, semi major axis, oblateness parameter, eccentricity in general and found that there is decrease in region of stability.

In this manuscript, the simulation technique is exploited to study the linear stability of the triangular equilibrium points around $\mathrm{L}_{4}$ by plotting $u(f)$ and $v(f)$. The The $u(f)$ and $v(f)$ function defines the solutions for the perturbed motion in the vicinity of $\mathrm{L}_{4}$. Figs 1- 10 shows $\mathrm{u}$ (f) and $\mathrm{v}$ (f) curve for different binary systems in the range $f \in[0,37.75 \pi]$. It is clear from the Figs. $1,3,5,7,9$ that the motion of the infinitesimal mass at the triangular points is oscillatory. It can be seen from Figs 2 and 10 that $u$ (f) versus $v$ (f) curve for Achird and $\mathrm{Xi}$ - Bootis shows almost the same behavior Similar nature can be seen for the binary systems Luyten, Alpha Cen AB, Kruger-60 which is obivous from Figs.4, 6 and 8. Also, Figs. 11-25 it is clear that oblateness which is considered apart from gravitational and radiation pressure of the primaries affects the trajectories of the infinitesimal mass. The change in oblateness parameter shows a significant change in the two-dimensional trajectory for the binary systems. The trajectory of the infinitesimal mass around the triangular points are plotted for three different values of oblateness parameters for the binary system. The threedimensional trajectories of the infinitesimal mass are shown in Figs.25-40 for the binary systems for different values of oblateness parameter which shows almost the same nature.

\section{References}

[1] Ammar MK (2008), the effect of solar radiation pressure on the Lagrangian points in the elliptical restricted three body problem, Astrophysics \& Space Science, 313, 393-408. http://dx.doi.org/10.1007/s10509-007-9709-z.

[2] Danby, JMA (1964), Stability of the triangular points in the elliptic restricted problem of three bodies, Astronomical Journal, 69, 165-172. http://dx.doi.org/10.1086/109254.

[3] Erdi B (2009), A parametric study of stability and resonance around L4 in the elliptical restricted three body problem, Celestial Mechanics and Dynamical Astronomy, 104,145- 158 http://dx.doi.org/10.1007/s10569-009-9197-2.

[4] Grebenikov EA (1964), On the stability of the Lagrangian Triangular Solutions of the Restricted Elliptic Three Body problem, Soviet Astronomy, 8(3), 451-459.
[5] Gyorgyey J (1985), on the nonlinear stability of motions around the elliptical restricted problem of three bodies, Celestial Mechanics and Dynamical Astronomy, 36(3), 281-285. http://dx.doi.org/10.1007/BF01230741.

[6] Halan PP \& Rana N (2001), The Existence and stability of equilibrium points in the Robe's restricted three body problem, Celestial Mechanics and Dynamical Astronomy, 79, 145-155. http://dx.doi.org/10.1023/A:1011173320720.

[7] Kumar S \& Ishwar B (2011) Location of collinear equilibrium points in the generalized photogravitational elliptical restricted three body problem, Int. Journal of Sciience \& Technology, 3(2), 157-162.

[8] Kumar V \& Choudhary RK (1986), On the stability of the triangular libration points for the photogravitational circular restricted problem of three bodies when both of the attracting bodies are radiating as well, Celestial Mechanics, 40(2), 155-170 http://dx.doi.org/10.1007/BF01230257.

[9] Markeev AP (1978), Libration points in celestial Mechanics and Cosmodynamics, nauka, Moscow, Russia.

[10] Markellos VV, Perdios E \& Labropoulou P (1992), Linear stability of the triangular equilibrium points in the phptogravitational elliptical restricted three body problem, Astrophysics \& Space Science, 194, $207-$ 213. http://dx.doi.org/10.1007/BF00643991.

[11] Narayan, A \& Singh.N (2014a), Motion and stability of triangular equilibrium points in elliptical restricted three body problem under the radiating primaries, Astrophysics \& Space Science, 352 (1) , 57-70. http://dx.doi.org/10.1007/s10509-014-1903-1.

[12] Narayan, A., Singh.N. (2014c), Resonance stability of triangular equilibrium points in elliptical restricted three body problem under the radiating primaries, Astrophysics \& Space Science, 353 (2) 441-455. http://dx.doi.org/10.1007/s10509-014-2085-6.

[13]Narayan, A \& Singh N (2014b), Stability of triangular lagrangian points in elliptical restricted three body problem under the radiating binary systems, Astrophysics \& Space Science, 353 (2) 457-464. http://dx.doi.org/10.1007/s10509-014-2014-8.

[14] Roberts G (2002), linear stability of the elliptic Lagrangian triangle solution in the three body problem, Differential equation, 182, 191218.

[15]Selaru D \& Cucu-Dumitrescu C (1995), Infinitesimal orbit around Lagrange points in the elliptic restricted three body problem , Celestial Mechanics and Dynamical Astronomy, 61(4), 333- 346. http://dx.doi.org/10.1007/BF00049514.

[16] Sharma, R.K., W.Frucke, G.Teleki. (1982), Sun and platenary system Reidel Publishing company, 435.

[17]Singh J \& Umar A (2012a), Motion in the photogravitational elliptic restricted three-body problem under an oblate primary, Astronomical Journal, 143, 109. http://dx.doi.org/10.1088/0004-6256/143/5/109.

[18]Singh J \& Umar A, (2012b), on the stability of triangular equilibrium points in the elliptic R3BP under radiating and oblate primaries, As$\begin{array}{lllll}\text { trophysics } \& \quad \text { Space } & \text { Science, } 341, & \end{array}$ http://dx.doi.org/10.1007/s10509-012-1109-3.

[19]Subbarao,P.V.,Sharma R.K.: 1975, A note on the stability of the triangular points of the equilibrium in the restricted three body problem, Astron. Astrophys., 43.

[20]Szebebely V (1967), Stability of the points of equilibrium in the restricted problem, Astronomical Journal, 72, 7-9. http://dx.doi.org/10.1086/110195.

[21]Zimvoschikov AS \& Thakai VN (2004), Instability of libration points and resonance phenomena in the photogravitational in the elliptical restricted three body problem, Solar System Research, 38 (2), 155-163. http://dx.doi.org/10.1023/B:SOLS.0000022826.31475.a7. 